S. Tsuruta ${ }^{(*)}$, and K. Nomoto ${ }^{(* *)}$

(*) Department of Physics, Montana State University

(**) Department of Earth Science and Astronomy, University of Tokyo, and Department of Physics, Brookhaven National Laboratory

\title{
INTRODUCTION
}

The observational evidence for the presence of dark matter is now generally accepted, with no lack of possible candidates (see e.g. Dekel, Einasto, and Rees 1986). The proposed candidates are devided into two groups, baryonic and non-baryonic. The latter is further devided to hot and cold dark matter. For the cold dark matter, among the first to be proposed is the axion. In this paper we shall not dwell on numerous cold dark matter candidates offered by particle physicists, for there are review articles on the subject (see e.g. Turner 1986, Primack 1986). The main purpose of the present report is to suggest that neutron star cooling theory and future space satellite programs (e.g. AXAF, XAO, LXAO) have a potential for offering the best astrophysical constraint on the axion mass and hence, giving valuable insight to some cosmological problems.

Even though other candidates for cold dark matter are perfectly plausible, axions may have the following advantages. Their presence was predicted, independently of cosmology, as a natural solution to the strong CP problem (Peccei and Quinn 1977). Also, once we accept their presence, we can carry out specific calculations, to predict various properties (e.g. their emissivities).

\section{CONSTRAINTS ON AXION MASS}

The axion is a relatively light, pseudoscalar boson associated with the Peccei and Quinn symmetry, a child of the strong CP problem and grandchild of the U(1) problem (Sato and Sato 1975, Weinberg 1978, Wilczek 1978). The symmetry breaking scale F, however, is left undetermined in the theory. The axion mass $\mathrm{m}_{\mathrm{a}}$ is related to the parameter $F$, roughly as $m_{a} \sim 10^{16} \mathrm{eV}^{2} / \mathrm{F}$ (e.g. Turner 1986).

The observational upper and lower limits to the value of $F$ could be set by cosmological and astrophysical considerations. The upper limit, $\mathrm{F} \sim 10^{12} \mathrm{GeV}$, which corresponds to $\Omega=1$, comes from cosmological constraints, that coherent axion oscillations in the presentday universe do not produce an unacceptably large energy density. It gives the lower limit to the axion mass, as $\mathrm{m}_{\mathrm{a}}>\sim 10^{-5} \mathrm{eV}$. The lower limit to $F$ (or upper limit to $m_{a}$ ) generally comes from stellar 
constraints, that various interactions in the stellar interiors involving axions would not be too fast to affect stellar evolution, to support observational evidence (Fukugita et al. 1982). The best stellar constraints to date, using the normal "invisible" axion model, are shown in Table 1 (Raffelt 1986a, b, Dearborn et al. 1986, ). We note that the constraints from cooling of white dwarfs and Helium burning stars are the most severe, both giving $\mathrm{m}_{\mathrm{a}}<\sim 0.01 \mathrm{eV}$.

It has been reported (Kim 1979,1984, Kaplan 1985) that axions should be even more "invisible" than proposed earlier. The important characteristics of this model, often referred to as the "hadronic model", is that these axions do not couple to leptons. The implication is that dominant axion emissivities in most stars (including all listed in Table 1) would be greatly reduced since their dominant axion interactions involve electrons.

\begin{tabular}{|c|c|c|}
\hline & $\mathrm{m}_{\mathrm{a}}(\mathrm{eV})$ & $F(G e V)$ \\
\hline Solar & $<\sim 3.2$ & $>\sim 3 \times 10^{6}$ \\
\hline $\begin{array}{c}\text { Red } \\
\text { Giants }\end{array}$ & $<\sim 0.06$ & $>\sim 2 \times 10^{8}$ \\
\hline $\begin{array}{c}\text { White } \\
\text { Dwarfs }\end{array}$ & $<\sim 0.01$ & $>\sim 10^{9}$ \\
\hline He Burning & $<\sim 0.01$ & $\sim 10^{9}$ \\
\hline
\end{tabular}

TABLE 1

Stellar constraints for "normal" axion model

The constraints for these "hadronic axions" are shown in Table 2 (Raffelt 1986 a). A major conclusion is that if this model is correct, the constraints on the axion mass from these stars are weakened enormously. Note that the strongest constraint is reduced to the axion mass of only a few eV (at least $10^{5}$ times larger than the lower limit corresponding to $\Omega=1$ ).

Another important implication of no axion interactions with electrons was emphasized by Kaplan (1985). That is, laboratory detecion of relic axions may become more difficult, if not impossible, than predicted earlier. Various laboratory experiments on detection of axions have been proposed (see e.g. a recent review by Smith 1986). It may be within our reach in a foreseeable future, to test by various laboratory experiments which of the invisible axion models, the "normal" or "hadronic", is correct. Therefore, it

\begin{tabular}{|c|c|c|}
\hline & $\mathrm{m}_{\mathrm{a}}(\mathrm{eV})$ & $\mathrm{F}(\mathrm{GeV})$ \\
\hline Solar & $<\sim 25$ & $>\sim 4 \times 10^{5}$ \\
\hline $\begin{array}{c}\text { Red } \\
\text { Giants }\end{array}$ & $<\sim 1.3$ & $>\sim 8 \times 10^{6}$ \\
\hline $\begin{array}{c}\text { Helium } \\
\text { Burning }\end{array}$ & $<\sim 2$ & $>\sim 6 \times 10^{6}$ \\
\hline
\end{tabular}

TABLE 2

Stellar constraints for "hadronic" axion model may be worthwhile to consider seriously an alternative possibility for offering a far stronger constraint on the axion mass. Here we propose that the combination of neutron star cooling theory and future X-ray satellite programs has a potential for offering such a possibility.

\section{AXION MASS LIMIT FROM NEUTRON STAR COOLING}

Since we can theoretically estimate the temperature of a neutron star of a given age through cooling theory, comparison of the theoretical temperature with observation will offer another means of 
stellar constraints on the axion mass, in the following sense. Any axion mass resulting in fast cooling (compared with observations) should be ruled out. At the moment the best obserational data come from the Einstein X-Ray Observtory (see e.g. Tsuruta 1986, Nomoto and Tsuruta 1986). Unfortunately, strictly speaking, several detected point sources in pulsars and supernova remnants offer only the upper limits to the stellar temperatures, mainly due to lack of spectroscopic resolution of the HRI which has the highest spacial resolution (Giacconi et al. 1979). However, there is a realistic possibility that future Xray satellites, especially AXAF, XAO and LXAO, could offer more conclusive data. Therefore, it may be worthwhile to explore what this option could do.

Among the point sources detected by the Einstein, RCW 103 is considered to be the best candidate as the detection of direct radiation from the neutron star surface (Tuohy and Garmire 1980, Tuohy et al. 1983). Therefore, we shall examine, in the following, what could be done by comparing neutron star cooling results with this source. If we assume that the RCW 103 data is the measurement of the stellar temperature, we can choose a cooling model which is consistent with this observational data. Then, models with very fast cooling agents such as quarks and pions should be ruled out for this particular source, and Case SX (with superfluid nucleons) of the FP neutron star model should be imposed (Tsuruta 1986). The FP model is a model of an intermediate stiffness constructed by Friedman and Pandaripande (1981), which is generally considered to be the most realistic nuclear model. (See Nomoto and Tsuruta 1987 for details). In the present calculations, the star cools with emission of axions, neutrinos and photons. Axion emissivities involving nucleons in the core are taken from Iwamoto 1984. Those involving electrons in the crust are taken from Itoh et al. 1986. The latter is drastically reduced for "hadronic axions". As noted earlier, this affects axion cooling of other stars drastically. However, this does not affect neutron star cooling, because the axion interaction with nucleons in the core, the dominant cooling process in a neutron star, is unaffected. Other input physics and methods are the same as in Nomoto and Tsuruta 1987.

We obtained neutron star cooling curvȩs for $F=10^{10}, 3 \times 10^{9}$, $10^{9}$, and $10^{8} \mathrm{GeV}$, corresponding to $\mathrm{m}_{\mathrm{a}} \sim 10^{-3}, 3 \times 10^{-3}, 0.0^{\prime} 1$, and 0.1 eV. These curves are compared with the Einstein data points. Assuming that the Einstein data for RCW 103 is the detection, the comparison of our theoretical curves with this source gives the most stringent upper limit to date: $\mathrm{m}_{\mathrm{a}}<\sim 10^{-3} \mathrm{eV}$ (Tsuruta and Nomoto 1986).

\section{CONCLUDING REMARKS}

If the Einstein data point for RCW 103 is confirmed to be the detection, neutron star cooling theory çan place the strongest upper limit to the axion mass, as $\mathrm{m}_{\mathrm{a}}<\sim 10^{-3} \mathrm{eV}$, with the corresponding $\Omega>\sim 0.01$. Then, the axion contribution to the dark matter should be at least as large as the baryonic contribution. The future space programs such as AXSF, XAO and LXAO should be crucial, with the realistic potential for confirming this detection. They may offer even more exciting outome, by the detection of other yet undiscovered sources. 


\section{ACKNOWLEDGEMENTS}

We thank Drs. M.J. Rees, K. Sato, M.A. Ruderman, G.. Raffelt, G. Börner, and D.E. Morris, for valuable discussions and comments. ST acknow ledges with thanks the hospitality of the directors of IOA in Cambridge and ISAS in Tokyo, where a part of this work was carried out. This work was supported in part by the NSF under grant AST-8602087, by MONTS under grant 16404216, and by DOA under contract DE-AC02$76 \mathrm{CH} 00016$.

\section{REFERENCES}

Dearborn, D.S.P., Schramm, D.N., and Steigman, G.: 1986, Phys. Rev. Lett. $56,26$.

Dekel, A., Einasto, J., and M. Rees: 1986, Rev. Mod. Phys., in press. Friedman, B., and Pandharipande, V.R.: 1981, Nucl. Phys. A361, 502. Fukugita, M., Watamura, S., and Yoshimura, M.: 1982, Phys. Rev. Lett. $\underline{48}, 1522$.

Giacconi, R. et al.: 1979, Astrophys. J. Lett. 230, 540 .

Iwamoto, N.: 1984, Phys. Rev. Lett. 53, 1198.

Itoh, N., et al. 1986: Private Communication.

Kaplan, D.N.: 1985, Nucl. Phys. B260, 215.

Kim, J.E.: 1979, Phys. Rev. Lett. 43, 10. : 1984, Seoul National Univ. Preprint SNUHE-84/02.

Nomoto, K., and Tsuruta, S: 1986, Astrophys. J. Lett. 305, L19. : 1987, Astrophys. J., Jan. 15, Vol. 312, in press.

Peccei, R.D., and Quinn, H.R.: 1977, Phys. REv. Lett. 38, 1440.

Primack, J.R.: 1986, Preprint SCIPP 86/65.

Raffelt, G.G.: 1986a, Ph.D. Thesis, Univ. of München. : 1986b, Phys. Rev. D, 33, 897, \& Phys. Lett. 166B, 402.

Sato, K., and Sato, H.: 1975, Prog. Theor. Phys. 54, 1564.

Smith,P.F.: 1986: Preprint RAL 86-029.

Tsuruta, S.: 1986, Com. Astrophys. $\underline{\mathrm{XI}}, 151$.

Tsuruta, S., and Nomoto, K.: 1986, in preperation.

Tuohy, I.R., and Garmire, G.P.: 1980, Astrophys. J. Lett. 239, L107.

Tuohy, I.R., Garmire, G.P., Manchester, R.N., and Dopita, M.A., 1983, Astrophys. J. 268, 778 .

Turner M.S.: 1986, Preprint.

Weinberg, S.: 1978, Phys. Rev. Lett. 444, 223.

Wilczek, F.: 1978, Phys. Rev. Lett. 40, 279.

\section{DISCUSSION}

CANIZARES: If there were some heating of the neutron star, say by accretion, wouldn't that make it impossible to determine an upper limit to the axion mass by your method?

TSURUTA: No, what you said does not apply in our case. Any possible heating mechanisms become important only for older neutron stars ( $t$ ? $\sim 10^{4}$ years) such as older radio pulsars or accreting neutron stars in 
a binary system (see e.g. Tsuruta 1979, Physics Reports, or Tsuruta 1986 in the text). However, exactly because of this, these objects were purposely avoided in our comparison. What I mean is that our objects for comparison are confined only to young, isolated neutron stars in supernova remnants (all with $t<\sim 10^{4}$ years) where accretion is not important. For instance, the object we specifically chose, RCW 103 , is only 1000 years old, and there is no possibility for its being an accreting binary star (see Tuohy et al.1983 in the text). For all of the objects of our choice, cooling definitely determines the stellar temperature, and therefore, our method works perfectly well (see Tsuruta and Nomoto 1986 in the text, for details)。 